Research Paper

\title{
Irisin Is Elevated in Skeletal Muscle and Serum of Mice Immediately after Acute Exercise
}

\author{
Julia Brenmoehl1, Elke Albrecht ${ }^{2}{ }^{\bowtie}$, Katrin Komolka², Lisa Schering ${ }^{2}$, Martina Langhammer ${ }^{3}$, Andreas \\ Hoeflich¹, Steffen Maak² \\ 1. Institute for Genome Biology, Leibniz Institute for Farm Animal Biology (FBN), Dummerstorf, Germany; \\ 2. Institute for Muscle Biology and Growth, Leibniz Institute for Farm Animal Biology (FBN), Dummerstorf, Germany; \\ 3. Institute for Genetics and Biometry, Leibniz Institute for Farm Animal Biology (FBN), Dummerstorf, Germany.
}

$\triangle$ Corresponding author: Dr. Elke Albrecht, Institute for Muscle Biology and Growth, Leibniz Institute for Farm Animal Biology. Wilhelm-Stahl-Allee 2, 18196 Dummerstorf, Germany. Tel: +49 38208 68858, Fax: +49 3820868852 E-mail: elke.albrecht@fbn-dummerstorf.de.

( ) Ivyspring International Publisher. This is an open-access article distributed under the terms of the Creative Commons License (http://creativecommons.org/ licenses/by-nc-nd/3.0/). Reproduction is permitted for personal, noncommercial use, provided that the article is in whole, unmodified, and properly cited.

Received: 2013.10.24; Accepted: 2014.02.24; Published: 2014.03.1I

\begin{abstract}
Recent findings regarding the response of fibronectin type III domain-containing protein 5 (Fndc5) and irisin to exercise are partly controversial. While the $25 \mathrm{kDa}$ form of Fndc5 can be observed in muscle and serum of different species, the $\sim 12 \mathrm{kDa}$ irisin band was not detectable up to now. The present study aimed to clarify whether irisin exists in its theoretical size of $\sim 12 \mathrm{kDa}$ in mice and if it is affected by exercise. Male mice were randomly assigned to a sedentary control group (CO), a group with free access to running wheels (RW), and a treadmill group (TM). Blood and leg muscles were collected to investigate the regulatory cascade including peroxisome proliferator-activated receptor gamma co-activator I-alpha (Ppargcla) and Fndc5. In western blot analysis, antibodies were used capable of differentiation between full-length Fndc5 and irisin. This enabled us to demonstrate that irisin exists in muscle and serum of mice independent of exercise and that it is increased immediately after acute exercise. Different transcripts of Ppargcla mRNA, but not Fndc5 mRNA, were up-regulated in the TM group. Furthermore, neither Fndc5 (25 kDa) nor Ppargc la protein was elevated in muscle tissue. The Ppargc la-Fndc5/irisin pathway did not clearly respond to mild exercise in the RW group. Our results provide evidence for the existence of irisin and for its immediate response to acute exercise in mice.
\end{abstract}

Key words: endurance exercise, Fndc5, irisin, myokine, Ppargc1a.

\section{Introduction}

Two years ago, Boström et al. (3) characterized irisin as a new myokine originating from cleavage of the transmembrane protein fibronectin type III domain-containing protein 5 (Fndc5). Mice overexpressing peroxisome proliferator-activated receptor gamma coactivator 1-alpha (Ppargc1a, also known as PGC1- $\alpha$ ) in muscle responded with an increase in Fndc5 mRNA. This increase could also be induced by physical exercise. It was demonstrated that recombinant Fndc5 induced a thermogenic program in white adipose tissue thus linking exercise with browning of adipose tissue indirectly (3). Cleavage and modifica- tion of Fndc5 was proposed as prerequisite for this action. However, cleavage of the extracellular part of Fndc5 and release of the resulting peptide irisin was only demonstrated in transfected HEK293 cells (3). Furthermore, Boström et al. (3) as well as subsequent studies employing western blot in tissues and serum of mice, rats, and humans $(24,28,32)$ failed to demonstrate a peptide of the size expected for irisin $(12 \mathrm{kDa})$, but analyzed proteins between 22 to $28 \mathrm{kDa}$ which corresponded to the size of full-length Fndc5.

Since the initial description of irisin, more than 25 ELISA (enzyme-linked immunosorbent assay) 
based studies were conducted in humans, mice, and pigs to elucidate either effects of exercise on circulating irisin $(9,13,17,18,20)$ or relationships between irisin level, body composition, type II diabetes and further diseases [reviewed by Novelle et al. (19)]. The results of these studies were controversial which may in part be caused by unspecific bindings of the capture antibody. Importantly, Erickson (8) stated that antibodies used in these ELISA kits must be validated by quantitative western blot to prove their specificity. Consequently, there is still no direct evidence for circulating irisin in any species (8).

The inducer of Fndc5/irisin - Ppargcla - was subjected to a detailed analysis recently (25). Expression of 4 different Ppargc1a transcripts (Ppargc1a1 to 4) was shown in a variety of murine tissues including skeletal muscle. Interestingly, specific expression of Ppargc1a4 in primary myotubes did not induce several classic Ppargcla1 targets but stimulated insulin-like growth factor 1 (Igf1) expression and suppressed expression of myostatin (25). The authors concluded from a series of subsequent experiments that forced Ppargc1a4 expression in muscle - either by transgenesis or by resistance training - may result in functional hypertrophy. The role of Ppargcla transcripts 2 and 3 was not further investigated in this study, however (25).

Against this background, we aimed to provide evidence for circulation of $12 \mathrm{kDa}$ irisin peptide in mice. Furthermore, we investigated effects of voluntary and acute exercise on circulating irisin, on expression of its precursor Fndc5, and on expression of different transcripts of its potential activator Ppargc1a.

\section{Material and Methods}

\section{Animals and husbandry}

All procedures were done in accordance to national and international guidelines and approved by our own institutional board (Animal Protection Board from the Leibniz Institute for Farm Animal Biology) as well as by the national Animal Protection Board Mecklenburg-Western Pomerania (file number: LVL-MV/TSD/7221.3-1.1-013/12).

We used the outbred mouse line DUhTP (Dummerstorf high treadmill performance) generated by selection for high treadmill performance for 117 generations as described elsewhere $(4,10)$, because mice are able to run long distances either in a treadmill or running wheel with sufficient variation. The animals were housed in Makrolon-cages Type II (EBECO, Castrop-Rauxel, Germany) in a semi-barrier system under environmentally controlled conditions with a $12 \mathrm{~h}$ light / $12 \mathrm{~h}$ dark cycle (room temperature
$22.5 \pm 0.2{ }^{\circ} \mathrm{C}$, humidity $\left.50-60 \%\right)$. Fresh tap water and fixed formula food for laboratory mice were supplied ad libitum (ssniff V1124: protein, $22 \%$; fat, $4.5 \%$; raw fiber, $3.9 \%$; ash, $6.8 \%$; ssniff Spezialdiäten, Soest, Germany).

\section{Running wheel and treadmill performance}

Three groups of mice were randomly assigned, each consisting of 11 to 12 70-days-old male DUhTP mice with $32.6 \pm 6.1 \mathrm{~g}$ mean body weight. One group (RW) was kept in cages including running wheels $(\mathrm{d}=$ $33.4 \mathrm{~cm}$ ) equipped with wheel counters (Tecniplast, Hohenpeissenberg, Germany) for a period of 3 weeks from the $4^{\text {th }}$ to the $70^{\text {th }}$ day of age. Another group of 70-days-old male mice (TM) was undertaken a submaximal test on a computer controlled treadmill. Details of the test were described elsewhere (10). Briefly, a special test program with increasing speed (start speed: $15 \mathrm{~m} / \mathrm{min}$, final speed: $38 \mathrm{~m} / \mathrm{min}$ ) was applied. The test stopped as soon as the mice remained at the stimulating equipment. Coeval mice without access to running wheels were used as control group $(\mathrm{CO})$.

Animals were sacrificed by decapitation at day 70 of age, TM mice immediately after the test, and serum samples were prepared according to standard procedures. Femoral muscles (quadriceps femoris), representative for a single, highly active leg muscle, and crus muscles (incl. soleus, extensor digitorum longus, and gastrocnemius), a group of heterogeneous muscles, were flash frozen within 15 min after death and stored in liquid nitrogen for subsequent analyses.

\section{Quantitative PCR (qPCR)}

Muscle tissue of 12 TM mice, 8 RW mice, and 8 CO mice was homogenized using the Xiril Dispomix (Xiril, Hombrechtikon, Switzerland) and Qiazol Lysis Reagent (Qiagen, Hilden, Germany) as described by the manufacturer. The RNA was isolated and purified with NucleoSpin Extract II reagent (Macherey-Nagel, Dueren, Germany) according to manufacturer's guidelines. RNA was quantified with a NanoDrop ND-1000 spectrophotometer (Peqlab, Erlangen, Germany). The RNA integrity was determined with an Experion RNA StdSens analysis chip (BioRad, Munich, Germany). All RNA samples had RQI numbers between 8.0 and 9.5. First strand cDNA was synthesized from 100 ng total RNA in $20 \mu$ reaction volume using iScript cDNA Synthesis Kit (BioRad) according to the provided protocol.

The iCycler MyiQ 2 with iQ detection system (BioRad) was used for qPCR as described in (1). Gene expression measurements were performed in duplicates in $10 \mu \mathrm{l}$ reaction volumes containing $10 \mathrm{ng}$ cDNA templates, $2 \mu \mathrm{M}$ of the respective forward and 
reverse primers, and $5 \mu 1$ SYBR Green Supermix (BioRad). The primers listed in Table 1 were selected from recent publications as indicated or designed with Primer 3 (Version 0.4.0., http://frodo.wi.mit. edu/primer3/) and synthesized by Sigma-Aldrich (Munich, Germany). The amplification involved a denaturation step $\left(95^{\circ} \mathrm{C}\right.$ for $3 \mathrm{~min}$ ) followed by 45 cycles $\left(95^{\circ} \mathrm{C}\right.$ for $10 \mathrm{~s}, 60^{\circ} \mathrm{C}$ for $30 \mathrm{~s}, 70^{\circ} \mathrm{C}$ for $\left.45 \mathrm{~s}\right)$. For amplification of Ppargcla transcripts 2 and 4, the annealing temperature was adapted to $62{ }^{\circ} \mathrm{C}$ and $58^{\circ} \mathrm{C}$, respectively. The specificity of the amplicons was analyzed by melting curve analysis. Crossing point $\left(\mathrm{C}_{\mathrm{p}}\right)$ values were determined automatically by iQ5 software (Version 2.1.97.1001, BioRad). For each qPCR, the amplification efficiency was calculated from a standard curve derived from six serial dilutions (1:1, $1: 10,1: 50,1: 100,1: 500,1: 1,000)$ as $\mathrm{E}=10^{-1 / \text { slope of standard }}$ curve -1 . The identity of the products was confirmed by sequencing (ABI PRISM 310 Genetic Analyzer; Applied Biosystems, Darmstadt, Germany). Results are expressed as fold-changes in experimental groups compared to a $\mathrm{CO}$ group (mice without running treatment). The expression values were normalized to beta-2-microglobulin [B2m, (1)] and hypoxanthin-phosphoribosyl-transferase 1 [Hprt1, (6)]. The significance of expression differences was calculated with the REST algorithm as described by Pfaffl [(22); REST 2009, Version 2.0.13, QIAGEN].

\section{Western immunoblotting}

Proteins were extracted from femoral muscles of 12 TM mice, 12 RW mice, and 11 CO mice and crus muscles from $12 \mathrm{TM}$ mice, $8 \mathrm{RW}$ mice and $8 \mathrm{CO}$ mice (app. $60 \mathrm{mg}$, each). Muscle tissue was homogenized in CelLytic MT lysis buffer (Sigma-Aldrich) supplemented with protease inhibitor cocktail (P8340, Sigma-Aldrich) using the Dispomix (Xiril). After centrifugation $\left(14,000 \mathrm{rpm}, 15 \mathrm{~min}, 4^{\circ} \mathrm{C}\right)$ and elimination of cell debris, protein content of the tissue lysate was quantified using the BSA mode of Nanodrop 1000 (Peqlab). Serum samples were measured directly by Nanodrop technology.

Twenty micrograms of muscle protein or $30 \mu \mathrm{g}$ protein of serum samples, respectively, diluted in Laemmli buffer with $B$-mercaptoethanol were separated on $15 \%$ sulfate-polyacrylamide gel electrophoresis gels and transferred to polyvinylidene difluoride (PVDF) membranes (Carl Roth, Karlsruhe, Germany). Equal loading of the gels and proper transfer of the proteins to the membranes were verified by Coomassie Blue staining according to standard procedures. We analyzed the protein expression of Ppargc1a, Fndc5, and irisin by using respective antibodies as follows. Membranes were blocked for $1 \mathrm{~h}$ in either $5 \%$ non-fat dry milk in Tris-buffered saline (TBS), for Fndc5 and Ppargc1a, or 1× Roti-Block (Carl Roth), for detection of irisin. Membranes were incubated with primary antibodies over night at $4{ }^{\circ} \mathrm{C}$. Horseradish peroxidase-conjugated goat anti rabbit IgG secondary antibody (Cell Signaling Technology, New England Biolabs, Frankfurt/Main, Germany) was diluted 1: 5,000 and incubated for $1 \mathrm{~h}$. Protein abundance was detected by enhanced chemiluminescence (TMA-100, Lumigen technology, Bioquote Limited, York, UK) using Kodak Image Station 4000 MM (Raytest, Straubenhardt, Germany). LabImage 1D software (Kapelan Bio-Imaging, Leipzig, Germany) was used to quantify intensity of specific bands. To verify the results, each blot, i.e. sample, was repeated 2 to 4 times. After exposure, the antibody was stripped from the membrane with stripping solution (Restore Plus Western Blot Stripping Buffer, Thermo Fisher Scientific, Schwerte, Germany) for $20 \mathrm{~min}$ at room temperature.

Table I: Sequences of primer sets used for amplification of specific cDNA.

\begin{tabular}{|c|c|c|c|c|c|}
\hline Locus & Primer & bp & Sequence 5'- 3' & Accession No. & Position \\
\hline B2m & forward & 175 & CCTGGTCTTTCTGGTGCTTG & NM_009735 & $69-89$ \\
\hline (reference) & reverse & & TTTCCCGTTCTTCAGCATTT & & $224-243$ \\
\hline Hprt1 & forward & 90 & TCCTCCTCAGACCGCTTTT & NM_013556 & $104-122$ \\
\hline (reference) & reverse & & CCTGGTTCATCATCGCTAATC & & $173-193$ \\
\hline \multirow[t]{2}{*}{ Fndc5 } & forward & 162 & CAACGAGCCCAATAACAACA & NM_027402.3 & $553-574$ \\
\hline & reverse & & AGAAGGTCCTCTCGCATTCTC & & $694-714$ \\
\hline Ppargcla & forward & 156 & GGACATGTGCAGCCAAGACTCT & NM_008904.2 & $148-169$ \\
\hline transcript $1^{\mathrm{A}}$ & reverse & & CACTTCAATCCACCCAGAAAGCT & & 281-303 \\
\hline Ppargcla & forward & 109 & CCACCAGAATGAGTGACATGGA & JX866946.1 & $19-40$ \\
\hline transcript $2^{\mathrm{A}, \mathrm{B}}$ & reverse & & GTTCAGCAAGATCTGGGCAAA & & $107-127$ \\
\hline Ppargcla & forward & 103 & AAGTGAGTAACCGGAGGCATTC & JX866947 & $94-115$ \\
\hline transcript $3^{\mathrm{A}}$ & reverse & & TTCAGGAAGATCTGGGCAAAG A & & $175-196$ \\
\hline Ppargcla & forward & 172 & TCACACCAAACCCACAGAAA & JX866948 & $687-706$ \\
\hline transcript $4^{\mathrm{A}}$ & reverse & & CTGGAAGATATGGCACAT & & $841-858$ \\
\hline
\end{tabular}

A Ruas et al. (25), B co-amplifies transcripts 2, 3, and 4. 
Primary antibody for Ppargc1a, (1: 1,000; sc-13067, Santa Cruz Biotechnology, Heidelberg, Germany) is supposed to detect isoform 1 and 4 with higher affinity and isoform 2 and 3 with lower affinity according to sequence homology. The Fndc5 antibody (1: 1,000; AP8746b, BioCat, Heidelberg, Germany) binds to the C-terminus of Fndc5 (amino acids [aa] 149-178, human) and detects a band of about $25 \mathrm{kDa}$, which is in the range of the calculated molecular weight for the protein. The specificity of binding was tested in parallel blots which were incubated with either the antibody or with the antibody previously blocked with the respective blocking peptide. The irisin antibody (1: 3,000; A00170-01-100, Biotrend, Cologne, Germany) was developed using full-length recombinant irisin and detects a $\sim 12 \mathrm{kDa}$ band of irisin and several additional bands of larger size. As a control for specific binding of the irisin antibody, again two membranes were processed in parallel, which were incubated with either the irisin antibody or with the irisin antibody previously blocked by incubation with full-length recombinant irisin (aa 32-143). The $\sim 12 \mathrm{kDa}$ band was blocked exclusively in that case.

\section{Immunohistochemical analysis}

Femoral muscle samples were cryo-sectioned (8 $\mu \mathrm{m}$ thick) using a Leica CM3050 S (Leica, Bensheim, Germany) cryostat microtome. Tissue sections were fixed with $4 \%$ paraformaldehyde and washed with PBS. Unspecific binding of the secondary antibody was blocked using $10 \%$ goat serum in PBS for $15 \mathrm{~min}$. Sections were incubated with the primary antibody against either irisin (1: 100) for $1 \mathrm{~h}$ at room temperature or Fndc5 (1: 100) at $4{ }^{\circ} \mathrm{C}$ overnight in a humidity chamber. Specific binding of primary antibody was detected with the respective goat anti rabbit IgG secondary antibody labeled with MFP 488 (MoBiTec, Goettingen, Germany). Nuclei were counterstained with $1 \mu \mathrm{g} / \mathrm{ml}$ Hoechst 33258 (Sigma-Aldrich). Slides were covered using Mowiol mounting medium including 1,4-diazabicyclo[2.2.2]octan (DABCO, Carl Roth) and appropriate cover-slips. Negative controls were incubated either omitting the primary antibody or blocking the primary antibody with the respective peptide. No unspecific binding of the secondary antibody and only minimal unspecific binding of the primary antibody was detected. Disturbing auto-fluorescence was reduced by including a step of Sudan black ( $0.1 \%$ in $70 \%$ ethanol) staining for 30 min prior to the blocking step. Immunofluorescence was visualized with a Nikon Microphot SA fluorescence microscope (Nikon, Duesseldorf, Germany) and an image analysis system equipped with $\mathrm{CELL}^{\wedge} \mathrm{F}$ software and a CC-12 high resolution color camera (OSIS, Muenster, Germany).

\section{Statistical analysis}

Statistical analysis was performed using the SAS statistical software (Version 9.2, SAS Inst., Cary, USA). Data were analyzed by ANOVA using the MIXED procedure with fixed factor group (treatment) and random animal. Values of different blots for the same tissue and antibody were included as repeated measurements. The t-test was used as post-hoc test with $P \leq$ 0.05 as threshold for significant differences.

Relationships between traits were calculated within groups as Pearson's-correlation coefficients using the CORR procedure of SAS.

\section{Results}

\section{Running performance of DUhTP mice}

Basic phenotypical and physiological parameters of the outbred line DUhTP were described elsewhere (4). Mice of the RW group, which had free access to the running wheel, ran on average $4.7 \pm 2.6 \mathrm{~km}$ per day during 3 weeks of voluntary endurance training. The body weight gain during this time was not significantly different $(P=0.11)$ from that of the $\mathrm{CO}$ group. However, running distance and body weight gain were negatively correlated $(r=-0.67, P=0.02)$ in the RW group. Mice subjected to a single submaximal test on a treadmill (TM group) ran $5.1 \pm 1.8 \mathrm{~km}$. They lost on average $2.0 \pm 1.3 \mathrm{~g}$ body mass during this test.

\section{Irisin is abundant in murine muscle and serum and is elevated after acute exercise}

To clarify whether the secreted cleavage product of Fndc 5 exists in mice, we used western blot analysis with an antibody specific to irisin. This antibody detects full-length recombinant irisin at about $12 \mathrm{kDa}$ (lane 6 in Fig. 1A, left) and also a $\sim 12 \mathrm{kDa}$ band in mouse serum and muscle tissue (lanes 2 to 5 in Fig. $1 \mathrm{~A}$, left). In serum samples, this band usually appeared as a doublet band. To test whether the slightly larger band was glycosylated irisin, PNGase treatment was applied to serum samples. The second band disappeared after this treatment (Fig. 1B). Several additional bands were still observed in muscle and serum. Therefore, recombinant irisin was used to block the specific binding of the antibody and to discriminate between specific and unspecific bands. The strong band of recombinant irisin disappeared when the antibody was blocked in advance. Accordingly, the bands of similar size detected in mouse serum (lanes 2 and 3 in Fig. 1A, right) and muscle (lanes 4 and 5) could also be blocked. Further bands of different sizes were considered as unspecific, because 
they were still detected by the primary antibody after blocking with recombinant irisin. Consequently, the chemiluminescence intensity of the $\sim 12 \mathrm{kDa}$ band was measured to elucidate the effect of acute or voluntary exercise on irisin level in serum and muscle of mice. Samples for comparison of irisin abundance were not treated with PNGase prior to analysis and the volume of the doublet band was measured.

Figure 2A shows a representative western blot of serum from each 3 mice of the 3 groups and the results of quantification for all mice. Mice of the TM group had higher serum irisin levels $(P<0.01)$ than $\mathrm{CO}$ or RW group mice. This was accompanied by higher irisin levels in homogenates of femoral $(P<$ $0.01)$ but not of crus muscles $(P>0.05$, Fig. $2 \mathrm{~B})$. There was no difference in irisin levels between $\mathrm{CO}$ and RW group $(P>0.5)$ neither in serum nor in muscle tissues. Using a serial dilution of recombinant irisin as a standard (7 dilutions 1:2, from $40 \mathrm{ng}$ to $0.625 \mathrm{ng}$ ), we estimated the basal concentration of irisin in serum to $1.4 \mu \mathrm{g} / \mathrm{ml}$ and the concentration after treadmill exercise to $2.7 \mu \mathrm{g} / \mathrm{ml}$. This corresponded to a 1.9-fold increase of circulating irisin.

To test whether the irisin abundance in serum is directly related to the distance covered during the test, Pearson correlation coefficients were calculated.
Neither irisin abundance in serum nor in muscle was directly associated with the running distance of either acute or voluntary exercise (data not shown).

\section{Expression of Fndc5 is not altered immediately after exercise}

Next we analyzed the irisin precursor Fndc5 at mRNA and protein level. The mRNA expression of Fndc5 was not different between CO, RW, and TM mice, neither in femoral nor in crus muscle tissue (Fig. $3 \mathrm{~A})$.

Fndc 5 protein was analyzed using an anti-Fndc5 antibody directed against the C-terminal end of the protein (corresponds to the murine Fndc5 aa 147-175), thus excluding the irisin peptide. This antibody recognized Fndc5 at the expected size of $\sim 25 \mathrm{kDa}$ in muscle (Fig. 3B, left). Specificity was confirmed by blocking the antibody with the respective peptide prior to incubation (Fig. 3B, right). In serum samples, Fndc5 was detected only in rare cases (data not shown). Therefore, Fndc5 was analyzed in muscle tissue only, separately for femoral and crus muscles. The protein abundance of Fndc5 was not altered by any type of exercise $(P=0.29)$ neither in femoral muscles nor in crus muscles (Fig. 3C).
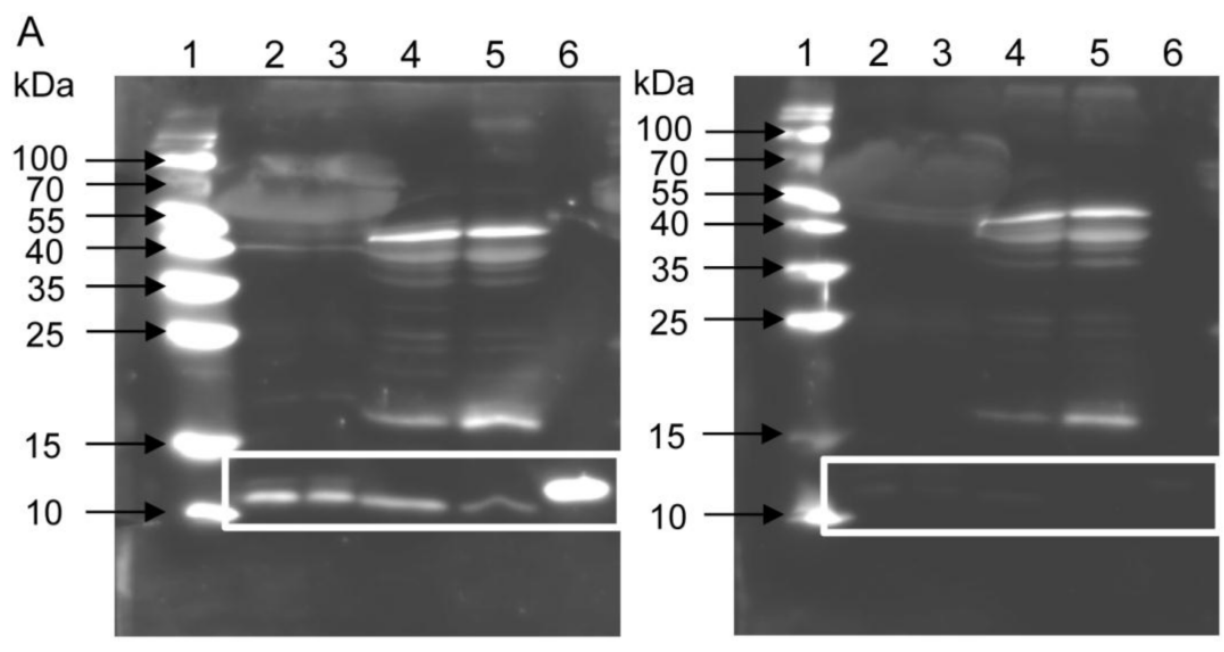

B

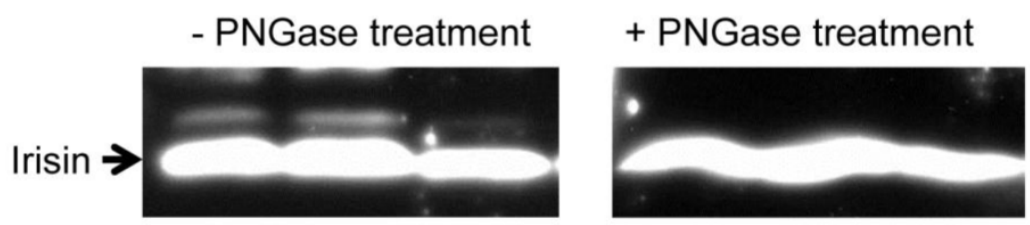

Figure I: Detection of irisin in mouse serum and muscle. (A) Western Blot of each 2 mouse serum (lane 2 and 3 ) and muscle samples (lane 4 and 5 ) and recombinant irisin (lane 6) incubated with either (left) antibody directed against irisin (aa 32-I43) or (right) antibody against irisin previously blocked with the recombinant protein. A specific band was detected at approximately $\sim 2 \mathrm{kDa}$ in serum and muscle tissue as indicated by the white frame. Images were identically enhanced in contrast. (B) Irisin band of mouse serum (left) without or (right) with PNGase treatment indicating that circulating irisin was partly glycosylated. Images were identically enhanced in contrast and cropped to the specific $\sim 12 \mathrm{kDa}$ band. 
A

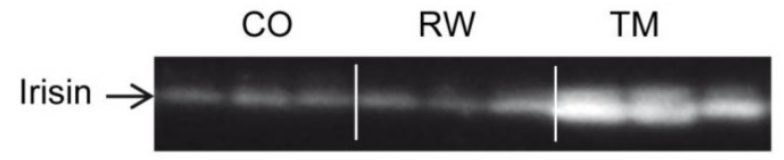

Serum

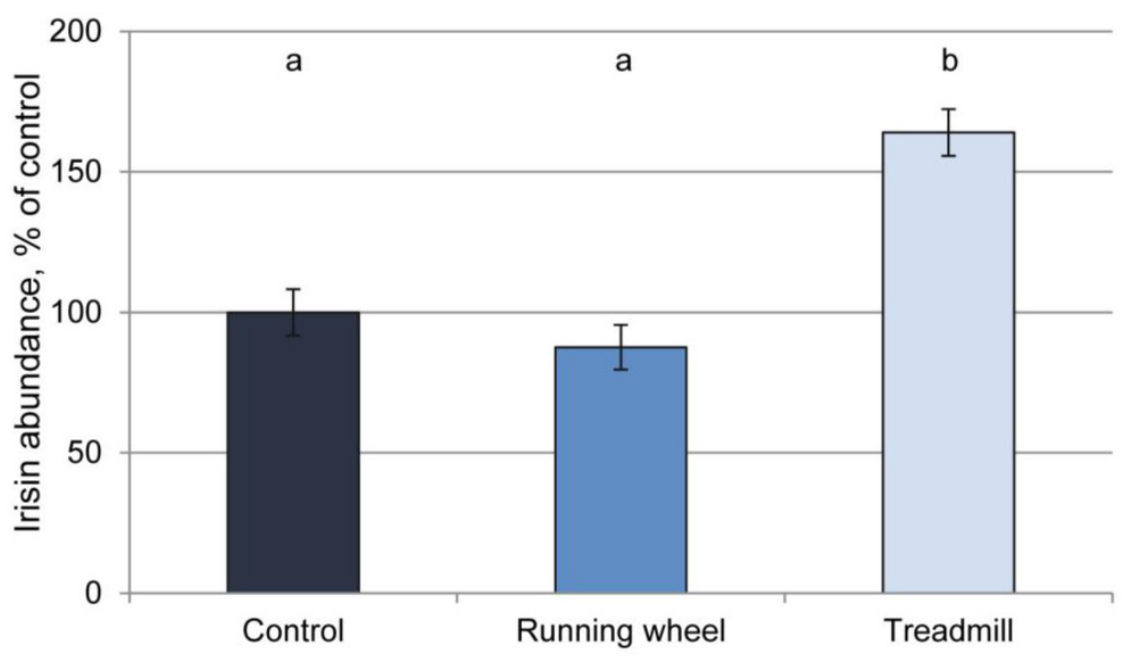

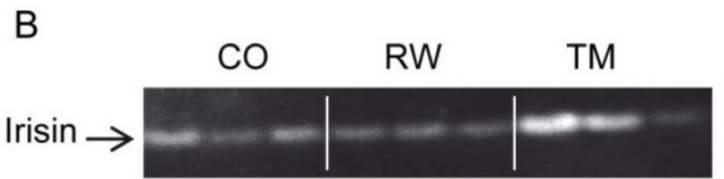

Femoral muscle tissue

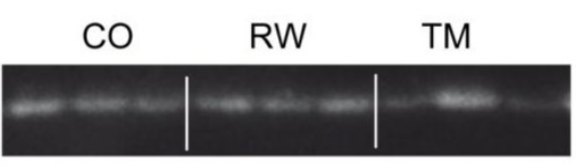

Crus muscle tissue

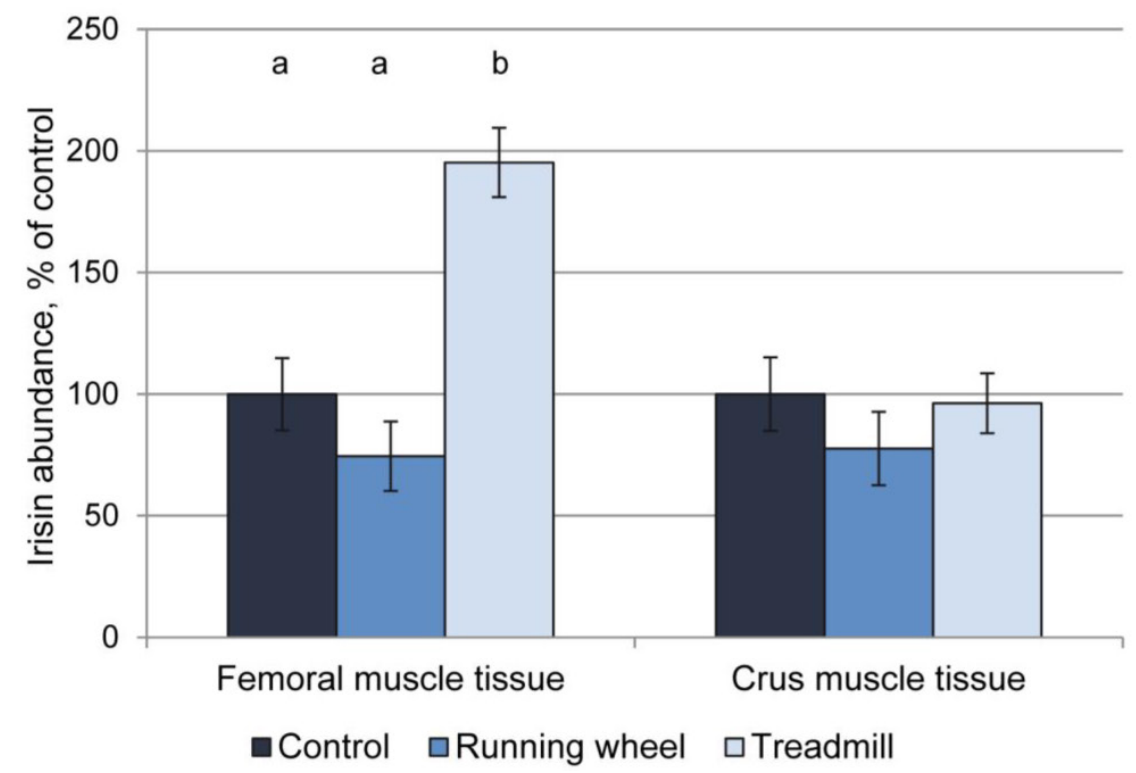

Figure 2: Protein abundance of irisin in (A) serum and (B) muscle tissue of mice after 3 weeks of voluntary exercise in a running wheel (serum and FM: $n$ $=12, C M: n=8)$ or one bout of treadmill exercise (serum, FM, and CM: $n=12$ ) relative to a sedentary control $($ serum and FM: $n=11, C M: n=8)$. Representative parts of the respective western blots for determination of irisin protein expression are shown above the graphs. Different letters indicate significant differences between groups $(P<0.05)$. CO - control group, RW - running wheel group, TM - treadmill group. 
A

Fndc5
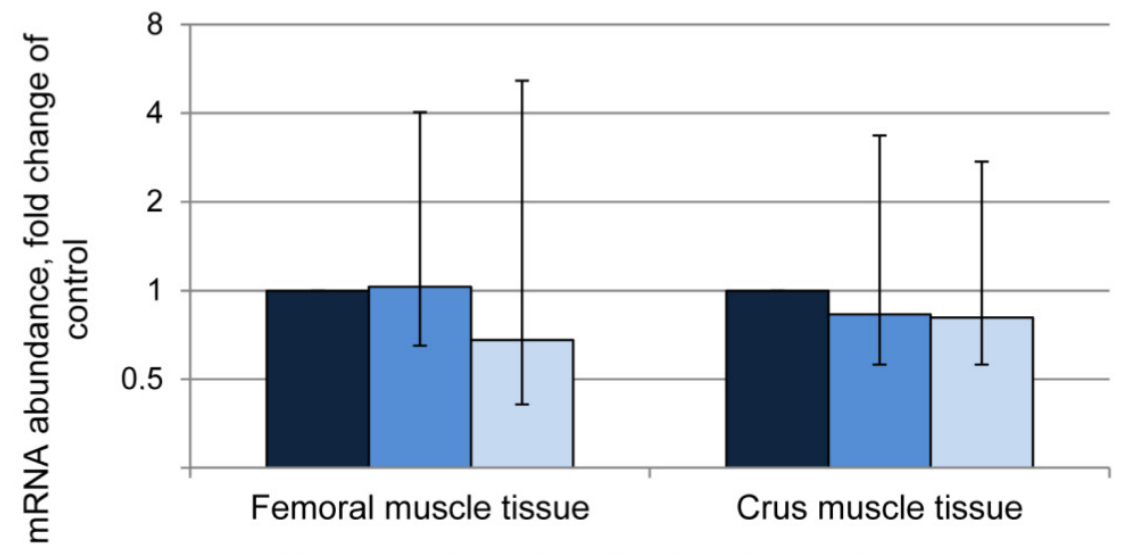

Femoral muscle tissue

Crus muscle tissue

घControl aRunning wheel 口Treadmill

B

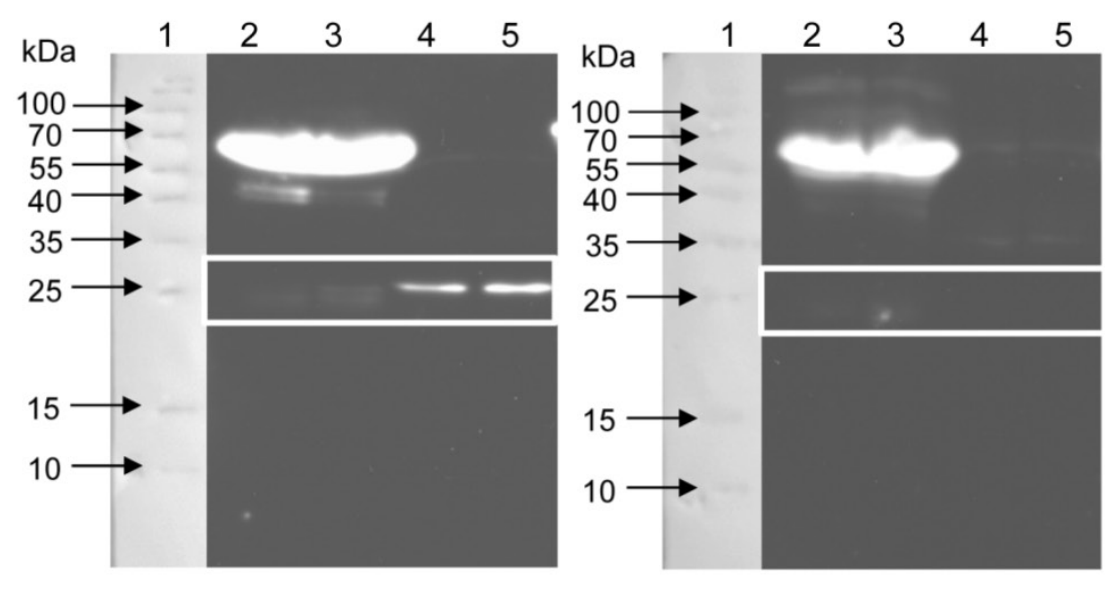

C
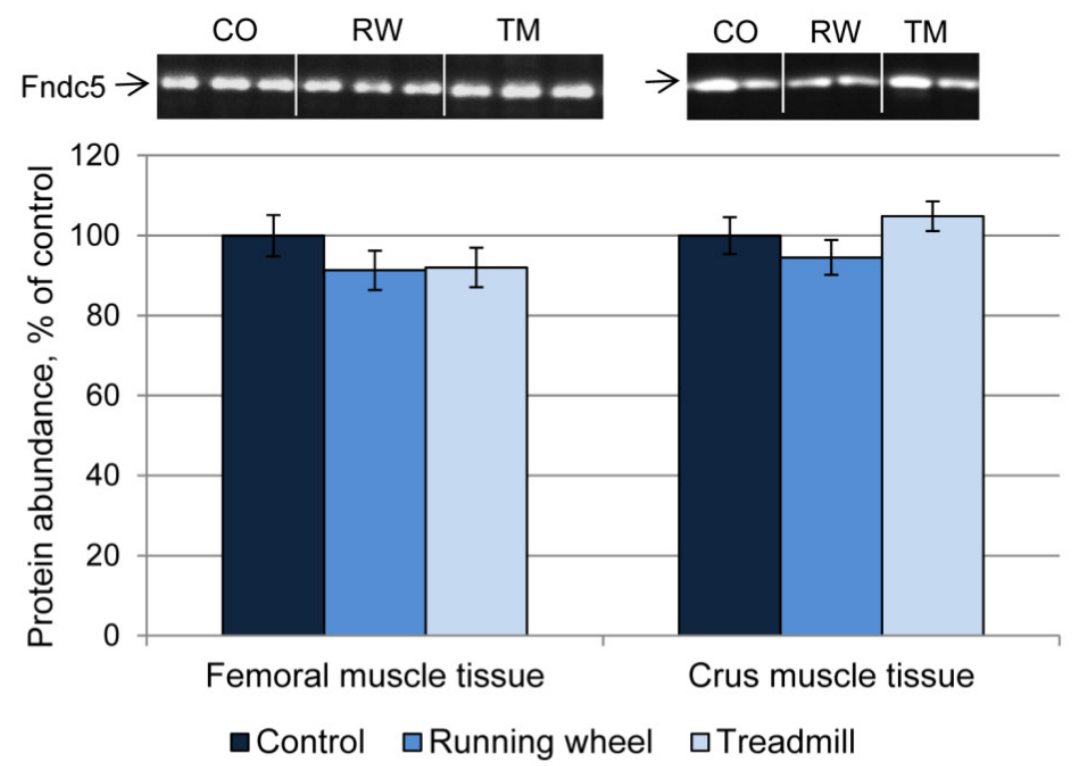

Figure 3: Abundance of Fndc5 mRNA and protein in femoral (FM) and crus muscle (CM) tissue of mice after 3 weeks of voluntary exercise in a running wheel (FM: $n=12, C M: n=8)$ or one bout of treadmill exercise (FM and CM: $n=12)$ relative to a sedentary control $(F M: n=11, C M: n=8)$. (A) mRNA abundance normalized to B2m and Hprtl. Bars represent means of fold changes compared to control group with $95 \%$ confidence intervals marked as vertical lines. (B) Western blot of each 2 mouse serum (lane 2 and 3 ) and muscle samples (lane 4 and 5) incubated with either (left) antibody directed against Fndc5 (aa 149-178) or (right) the antibody against Fndc5 previously blocked with the corresponding blocking peptide. The marker lane is laid on the image to determine the protein size. A specific band was detected at $\sim 25 \mathrm{kDa}$ exclusively in muscle tissue as indicated by the white frame. Images were identically enhanced in contrast. (C) Protein abundance of Fndc5 determined by quantitative western blot analysis. Representative parts of respective western blots are shown above the graph. CO - control group, RW - running wheel group, TM - treadmill group. 


\section{Immunohistochemical detection of irisin in muscle tissue indicates extracellular localization}

The cellular localization of irisin and its precursor Fndc5 was visualized and studied in femoral muscle tissue. Fndc5 was detected at the sarcolemma as expected for a transmembrane protein and additionally in the sarcoplasm showing a punctate signal, but not in the intercellular space between muscle fibers (Fig. 4A). Additionally, few cells located in connective tissue showed immunohistochemical Fndc5 staining at the cell membrane. This observation was also made in mice from $\mathrm{CO}$ and $\mathrm{RW}$ groups (data not shown).

Irisin-related immunofluorescence was detected as weak, diffuse signal across muscle fibers and was more intense at muscle fiber membranes and between muscle fibers (Fig. 4B) in TM mice. Weaker but similarly located signals for irisin were observed in $\mathrm{CO}$ and RW mice (data not shown).

\section{Ppargcla mRNA but not protein increases immediately after acute exercise}

To elucidate the proposed induction of Fndc5/irisin by Pparga1a, we then investigated mRNA and protein expression of different transcripts and isotypes of Ppargc1a in skeletal muscle. Using primers published by Ruas et al. (25), 3 of 4 transcripts could be analyzed separately (transcripts 1, 3, and 4). The primers for transcript 2 described by Ruas et al. (25) co-amplified transcripts 3 and/or 4 as indicated by double peaks in the melting curve analysis resulting from different amplicon lengths. An in silico-analysis of all described transcripts revealed that a separate analysis of transcript 2 with standard qPCR techniques is precluded by its identity to transcript 3 .
It differs from transcript 2 only by a 70 bp longer exon 1. Consequently, Ppargc1a2 could not be considered further in the present study.

The abundance of transcript 4 was 9- and 6-fold higher in the TM compared to the $\mathrm{CO}$ group in femoral and crus muscles, respectively (Fig. 5A, B). There was a positive relationship $(r=0.63$ and $0.69, P<0.05)$ between Ppargcla4 mRNA abundance and running performance in the TM group (Fig. 5C) in femoral and crus muscles, respectively. Surprisingly, the strongest increase in mRNA abundance after treadmill exercise was measured for transcript 3 . Mice of the TM group had 33- and 10-fold higher Ppargc1a3 mRNA expression than the sedentary $\mathrm{CO}$ group in femoral and crus muscles, respectively (Fig. 5A, B). In contrast to transcript 4 , there were no significant relationships between individual increase in mRNA abundance and running distance. Voluntary exercise in a running wheel (RW group) had no effect on Ppargc1a3 and 4 mRNA abundances in both muscle groups. Transcript 1 of Ppargcla was slightly but significantly up-regulated in TM mice (1.6- and 1.9-fold, in femoral and crus muscles, respectively, $P<0.01$ ) and also in femoral muscle of RW group (1.5-fold, $P=0.025$ ).

Next, the consequences of exercise on protein abundance of Ppargcla were investigated. The antibody against Ppargcla is supposed to detect different isoforms. Western blot analysis revealed bands of different size, usually larger than the predicted sizes of the respective isoforms (Ppargc1a1: $\sim 90 \mathrm{kDa}$; Ppargc1a2 and 3: $41 \mathrm{kDa}$; Ppargca1a4: $29 \mathrm{kDa}$, Uni-Prot O70343). Separate analysis of each band revealed similar protein amounts $(P>0.05)$ between $\mathrm{CO}$ and treatment groups, in both femoral and crus muscles. The results for isoform 4 are exemplarily shown in Figure 6.
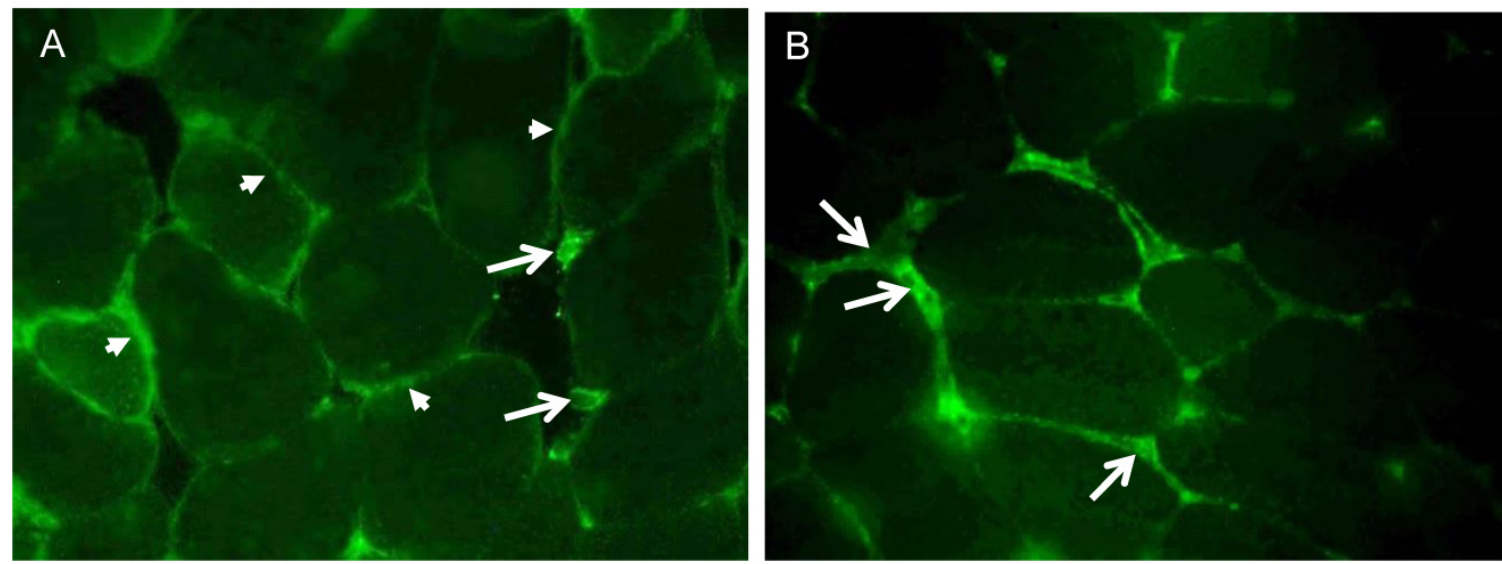

Figure 4: Cellular localization of (A) Fndc5 and (B) irisin in muscle cross sections of rectus femoris of a treadmill exercised mouse. Cryo-sections were immunostained with anti-Fndc5 and irisin primary antibodies and a MFP488 labeled goat anti-rabbit lgG secondary antibody. Fndc5 immunoreactivity was detected at the muscle fiber membrane (A, arrowheads) and punctuate in the cytoplasm, as well as in additional cells in the connective tissue (A, arrows). Irisin was mainly located in the intercellular space (B, arrows). 
A

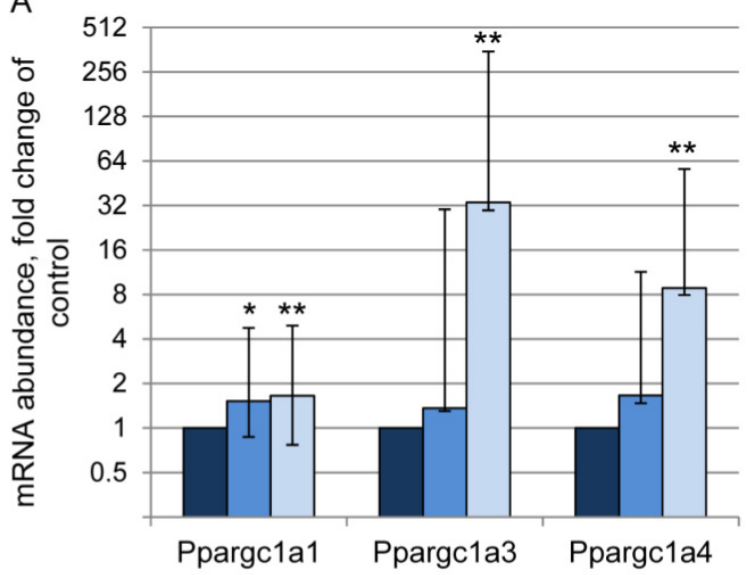

B

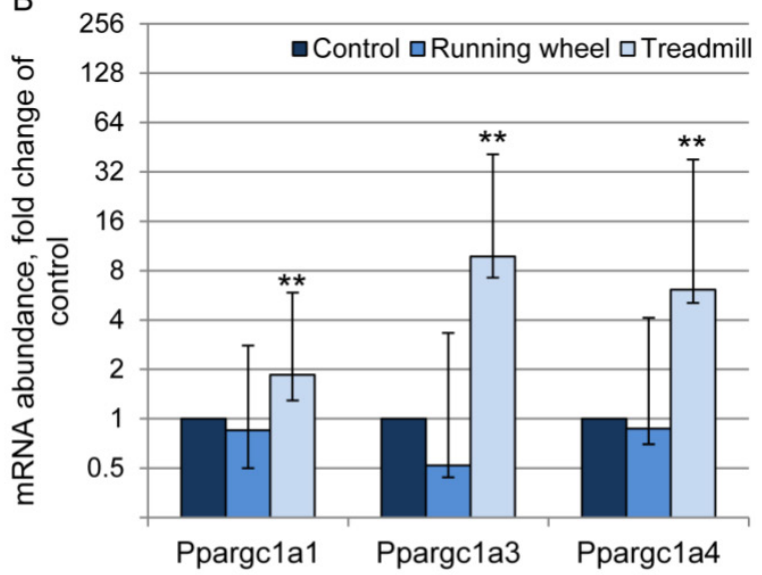

$\mathrm{C}$

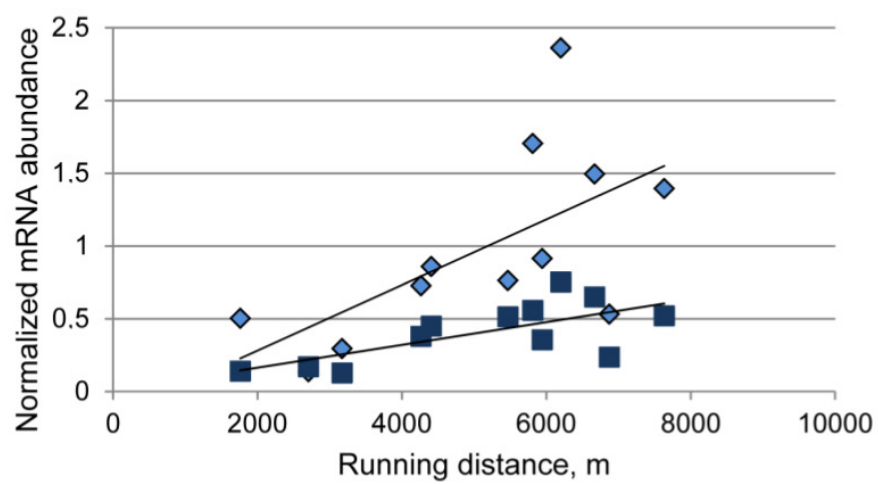

$\diamond$ Femoral muscle tissue $\square$ Crus muscle tissue

Figure 5: Relative mRNA expression of Ppargcla transcripts I, 3, and 4 in femoral (A) and crus (B) muscle tissue of mice after 3 weeks of voluntary exercise in a running wheel $(n=8)$ or one bout of treadmill exercise $(n=12)$ compared to a sedentary control $(n=8)$. The values were normalized to $B 2 m$ and Hprtl. Bars represent means of fold changes compared to control group with $95 \%$ confidence intervals marked as vertical lines. Asterisks indicate significant differences to control $(* P<0.05$, $* *<<0.01)$. (C) Relationship between Ppargcla4 mRNA abundance, normalized to Hprt I $\left(2^{-\Delta C_{p}}\right)$, in femoral and crus muscles of mice and running distance during one bout of treadmill exercise $(r=0.63$ and 0.69 , respectively, $P<0.05)$.
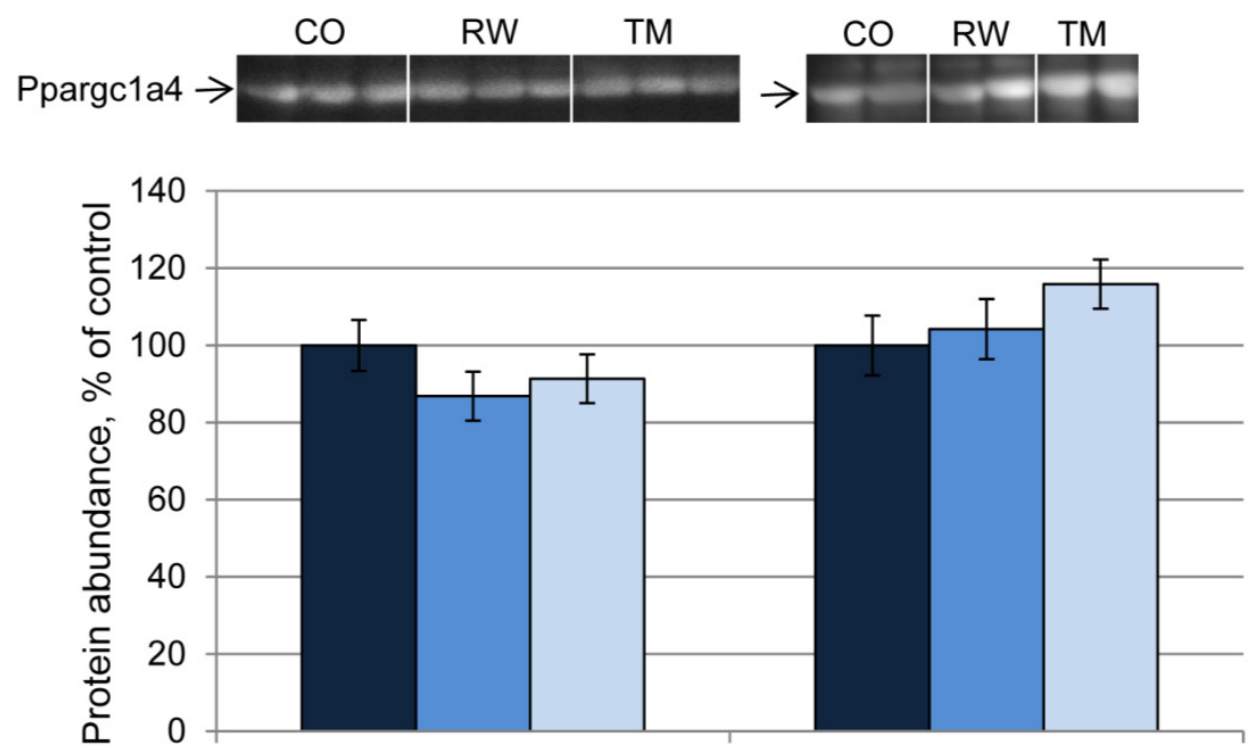

Femoral muscle tissue

Crus muscle tissue

Control aRunning wheel 口Treadmill

Figure 6: Protein abundance of Ppargcla4 in femoral (FM) and crus muscle (CM) tissue of mice after 3 weeks of voluntary exercise in a running wheel (FM: $n=12, C M: n=8)$ or one bout of treadmill exercise (FM and CM: $n=12$ ) relative to a sedentary control (FM: $n=I I, C M: n=8)$. Representative parts of respective western blots are shown above the graph. $\mathrm{CO}$ - control group, RW - running wheel group, TM - treadmill group. 


\section{Discussion}

In the present study, we investigated the Ppargc1a-Fndc5/irisin response in mice long-term selected for high treadmill performance (DUhTP) after a single bout of treadmill exercise or 3 weeks of voluntary exercise in a running wheel compared to a sedentary control. Mice of this line were selected because of their improved running capabilities with sufficient variation in running distances. The genetically fixed high running capacity of these mice was not achieved by training and the voluntary exercise activity of DUhTP mice was not distinct from that in unselected control mice (4).

In this mouse model, we demonstrated that irisin, the cleavage product of Fndc5, was present in skeletal muscle tissue and serum under resting conditions. Immunohistochemical analysis confirmed presence of irisin in murine skeletal muscle tissue. Its predominant location in the extracellular space between muscle cells is in agreement with the proposed mechanism of cleavage of the extracellular domain of transmembrane protein Fndc5 (3).

This is the first study that detected irisin by western blot at its predicted size of $\sim 12 \mathrm{kDa}$ and quantified exercise induced changes of this peptide in mice. Irisin responded with a significant, immediate increase to acute but not to repeated voluntary exercise in femoral muscle and in circulation. A similar reaction of irisin was observed in unselected mice (unpublished data) thus excluding a restriction of the results to the model used here. The increase of irisin in femoral but not in crus muscles after treadmill running may correspond to higher exposure of femoral muscles. Likewise, force measurements have shown that e.g. the gastrocnemius muscle, which is contained in crus muscle tissue, is activated only with increasing speed (18).

Our results of the blocking experiment provided evidence for the specific detection of irisin at $\sim 12 \mathrm{kDa}$. The used antibody is directed against aa 32-142 of human Fndc5 thus covering the irisin peptide completely. In contrast, Roca-Rivada et al. (24) failed to detect a quantifiable $12 \mathrm{kDa}$ irisin band in the secretome of rat skeletal muscle using an antibody recognizing aa 42-112 of human Fndc5. Obviously, binding to all amino acids of irisin is crucial for an efficient detection of the peptide. Numerous further studies employing western blot to detect Fndc5 and/or irisin used antibodies recognizing aa 149-178 of the human protein $(3,27-30,33)$. Importantly, this antibody cannot detect irisin per se as it is directed against the C-terminus of Fndc5 which does not include any sequence from the irisin peptide $(8,23,24)$. Accordingly, full length Fndc5 protein was detected at $\sim 25 \mathrm{kDa}$ with this antibody in our study.

Irisin appeared in serum as two bands at $\sim 12$ and $\sim 13 \mathrm{kDa}$ corresponding to the native and the glycosylated peptide, respectively, as confirmed by PNGase treatment. This is in contrast to a study on recombinant irisin which was detected as bands of 15 $\mathrm{kDa}$ (native), $22 \mathrm{kDa}$ (one site glycosylated) and 25 $\mathrm{kDa}$ (two sites glycosylated) as predominant form in a specific yeast expression system (33). It remains to be investigated whether the size discrepancy for glycosylated irisin is due to different experimental settings in both studies.

To elucidate the background of the exercise induced irisin increase we investigated the proposed upstream factors, Ppargc1a and Fndc5 $(3,27)$, in the same mice at mRNA and protein level. Additionally, recent findings on expression of Ppargc1a isoforms in skeletal muscle were considered (25). A transcript specific analysis of Ppargcla mRNA abundance revealed a significant induction of transcripts 1,3 , and 4 by acute exercise in murine femoral and crus muscle tissue. In contrast, voluntary wheel running did only result in a 1.5-fold increase of transcript 1 in femoral muscle. Boström et al. (3) observed a $\sim 2$-fold up-regulation of total Ppargcla mRNA in response to wheel running. This was likely caused by transcript 1 according to our data. Transcript 4 increased 6- to 9-fold in skeletal muscle of TM mice, whereas Ruas et al. (25) noticed a 3-fold increase in human Ppargc1a4 only when resistance and endurance training were combined. In our experiment Ppargcla3 mRNA responded with the largest increase to acute exercise in femoral and crus muscles (33- and 10-fold up-regulation, respectively). Chinsomboon et al. (5) reported a 125-fold increase of a Ppargcla transcript resulting from an alternative promoter in exercised mice. This promoter however, is responsible for transcription of Ppargc1a2, 3, and 4 as was shown later (25). Consequently, our results provide evidence that transcript 3 contributes a large proportion to the exercise-induced increase in total Ppargcla mRNA. The role of transcript 2 remains to be investigated. Importantly, transcripts 1,3 , and 4 regulate different gene programs (25). Transcript 1 affects numerous classical Ppargc1a targets whereas transcript 4 exerts effects on IGF1 and myostatin signaling. The observed correlation between Ppargcla4 mRNA abundance and individual running distance may thus indicate a link between physical activity and IGF1/myostatin signaling. Ppargcla transcript 3 was shown to affect 69 genes which await further investigation (25). Collectively, our results support the notion that Ppargcla transcripts are inducible by exercise to a different extent. Whether the slight increase of transcript 1 after both types of exercise mediates long term effects of 
exercise in general and the strong up-regulation of transcripts 3 and 4 immediately after acute exercise is responsible for fast reaction of skeletal muscle to exercise, remains to be addressed in further studies. In support of this assumption, Perry et al. (21) described a "saw tooth-shaped" Ppargcla mRNA increase with peaks immediately after single training bouts in humans using primers supposed to amplify all human transcripts. In contrast, Ppargcla protein was elevated with several hours delay and accumulated with increasing number of exercise bouts. This may explain our observation of unchanged Ppargcla protein in mice immediately after acute exercise.

Boström et al. (3) demonstrated that Fndc5 mRNA was induced by overexpression of Ppargcla and that this increase in Fndc5 could also be observed after physical exercise in human and mouse. The positive effect of exercise on Fndc5 expression was confirmed in a number of studies at mRNA $(11,15,18,24)$ and protein level $(24,29)$ in different experimental settings in rats and humans. We detected Fndc5 at 25 $\mathrm{kDa}$ in femoral as well as in crus muscles with western blot and localized the protein immunohistochemically, in accordance with Dun et al. (7), mainly at muscle cell membranes as expected for a transmembrane protein. In our experiment however, Fndc5 mRNA as well as protein remained unchanged in both muscle tissues after voluntary and immediately after acute exercise. This is in line with several studies which did not find a clear response of Fndc5 mRNA and/or protein to physical exercise in humans and pigs $(2,9,14,20,31)$ or exercise mimetics in cell cultures (26). Studies in rats, however, support the notion that there is a relationship between the abundance of Fndc5 and the oxidative capacity of muscle. Stephenson et al. (29) reported on elevated Fndc5 associated with higher oxidative capacity in glycolytic muscle of rats with higher running ability. Roca-Rivada et al. (24) described a $40 \%$ higher Fndc5 secretion from oxidative compared to glycolytic muscle under basal conditions and a significant increase after 3 weeks of voluntary wheel running in both muscles. Beside glycolytic m. quadriceps femoris from upper hind leg, we have analyzed crus muscles from lower hind leg comprising different muscles with different metabolic fiber types. Given the equal sampling of all mice in the experimental groups and the results obtained in rat muscles, we would have been able to detect changes in Fndc5 protein if present even in this heterogeneous muscle preparation.

In contrast to other studies, we observed Fndc5 protein only in single cases in serum of mice. In humans, Huh et al. (11) reported high levels of circulating Fndc5 $(25 \mathrm{kDa})$ in a subpopulation of young athletes and hypothesized that this might have resulted from muscle damage rather than being a physiological response. In conclusion, the reaction of Fndc5 to physical exercise remains controversial. Time series analyses with defined exercise regimens on defined muscles could resolve the controversies.

We analyzed the proposed Ppargc1a - Fndc5 irisin signaling chain $(3,27)$ at mRNA and protein level in response to acute exercise. The finding that only the first (Ppargcla mRNA) and the last (circulating irisin peptide) chain link were elevated in acute response to exercise indicates that additional, unknown mechanisms for irisin secretion from muscle may exist. Nevertheless, fast induction of Ppargcla mRNA upon exercise may start a process of recovering muscle irisin and its precursor Fndc5. The relatively high basal level of irisin suggests that irisin cleavage and secretion is a continuous, physiological process in mice. This is remarkably different from observations in other species. A recent study provided evidence for a different genetic make-up of the human Fndc5 locus resulting in drastically reduced expression of the full-length protein in vitro. Consequently, it was supposed that irisin cannot be produced by cleavage of Fndc5 and seems not to exist in humans at all (23). Own results in cattle demonstrated that full-length Fndc5 is abundant in skeletal muscle but irisin is neither detectable in muscle nor in circulation under resting conditions (12). Since direct evidence for existence of irisin was not yet provided in any species except in mice of this study, it is necessary to target possible species specificities in further investigations.

\section{Conclusions}

For the first time, our study provides evidence for the existence of irisin as $\sim 12 \mathrm{kDa}$ peptide in muscle tissue and for its circulation in mice under basal conditions. Muscle and circulating irisin increased immediately after acute but not after repeated voluntary exercise. Since this increase was not paralleled by an induction of Ppargc1a protein and Fndc5 mRNA and protein it is likely that the acute irisin response is mediated by additional, unknown factors. The elevated mRNA abundance of different Ppargcla transcripts after acute exercise however, may indicate that Ppargc1a induces recovery of muscular Fndc5 and irisin. The mRNA abundance of Ppargcla4 was positively related to running distance and may thus be a marker for exercise capacity. Collectively, these new findings extend the knowledge on irisin and may provide a basis to revisit the Ppargcla-Fndc5/irisin signaling pathway under consideration of species specificities. 


\section{Abbreviations}

aa: amino acid; B2M: beta-2-microglobulin; CO: control; $\mathrm{C}_{\mathrm{p}}$ : crossing point; DUhTP: Dummerstorf high treadmill performance; ELISA: enzyme-linked immunosorbent assay; Fndc5: fibronectin type III domain-containing protein 5; Hprt1: hypoxanthin-phosphoribosyl-transferase 1; IGF1: insulin-like growth factor 1; Ppargcla: peroxisome proliferator-activated receptor gamma coactivator 1-alpha; qPCR: quantitative polymerase chain reaction; $\mathrm{RW}$ : running wheel; TBS: Tris-buffered saline; TM: treadmill.

\section{Acknowledgement}

We are grateful to L. Chau, S. Hinrichs, E. Schwitulla, S. Foß, S. Geist, B. Lucht, S. Alm, and K. Ullerich for expert technical assistance and animal care.

This work was supported in part by grants of the Leibniz Association (grant no. SAW-2013-FBN-3) and the Deutsche Forschungsgemeinschaft (grant no. DFG H0 2003/6-1).

\section{Competing Interests}

The authors have declared that no competing interest exists.

\section{References}

1. Albrecht E, Komolka K, Kuzinski J, Maak S. Agouti revisited: Transcript quantification of the ASIP gene in bovine tissues related to protein expression and localization. PLoS One. 2012; 7: e35282.

2. Besse-Patin A, Montastier E, Vinel C, Castan-Laurell I, Louche K, Dray C, Daviaud D, Mir L, Marques MA, Thalamas C, Valet P, Langin D, Moro C, Viguerie N. Effect of endurance training on skeletal muscle myokine expression in obese men: identification of apelin as a novel myokine. Int J Obes (Lond). 2013; doi: 10.1038/ijo.2013.158.

3. Boström P, Wu J, Jedrychowski MP Korde A, Ye L, Lo JC, Rasbach KA, Boström EA, Choi JH, Long JZ, Kajimura S, Zingaretti MC, Vind BF, Tu H, Cinti S, Højlund K, Gygi SP, Spiegelman BM. A PGC1-a-dependent myokine that drives brown-fat-like development of white fat and thermogenesis. Nature. 2012; 481: 463-468.

4. Brenmoehl J, Walz C, Renne U, Ponsuksili S, Wolf C, Langhammer M, Schwerin M, Hoeflich A. Metabolic adaptations in the liver of born long-distance running mice. Med Sci Sports Exerc. 2013; 45: 841-850.

5. Chinsomboon J, Ruas J, Gupta RK, Thom R, Shoag J, Rowe GC, Sawada N, Raghuram S, Arany Z. The transcriptional coactivator PGC-1alpha mediates exercise-induced angiogenesis in skeletal muscle. Proc Natl Acad Sci U S A. 2009; 106: 21401-21406.

6. de Kok JB, Roelofs RW, Giesendorf BA, Pennings JL, Waas ET, Feuth T, Swinkels DW, Span PN. Normalization of gene expression measurements in tumor tissues: comparison of 13 endogenous control genes. Lab Invest. 2005; 85: 154-159.

7. Dun SL, Lyu RM, Chen YH, Chang JK, Luo JJ, Dun NJ. Irisin-immunoreactivity in neural and non-neural cells of the rodent. Neurosci. 2013; 240: 155-162.

8. Erickson HP. Irisin and FNDC5 in retrospect: An exercise hormone or a transmembrane receptor? Adipocyte. 2013; 2: 289-293.

9. Fain JN, Company JM, Booth FW, Laughlin MH, Padilla J, Jenkins NT, Bahouth SW, Sacks HS. Exercise training does not increase muscle FNDC5 protein or mRNA expression in pigs. Metabolism. 2013; 62: 1503-1511.

10. Falkenberg H, Langhammer M, Renne U. Comparison of biochemical blood traits after long-term selection on high or low locomotory activity in mice. Arch Tierz. 2000; 43: 513-522.

11. Huh JY, Panagiotou G, Mougios V, Brinkoetter M, Vamvini MT, Schneider BE, Mantzoros CS. FNDC5 and irisin in humans, I. Predictors of circulating concentrations in serum and plasma and II. mRNA expression and circulating concentrations in response to weight loss and exercise. Metabolism. 2012; 61: $1725-1738$.
12. Komolka K, Albrecht E, Schering L, Brenmoehl J, Hoeflich A, Maak S. Locus characterization and gene expression of bovine FNDC5: Is the myokine irisin relevant in cattle? PLoS One. 2014; 9: e88060.

13. Kraemer RR, Shockett P, Webb ND, Shah U, Castracane VD. A Transient Elevated Irisin Blood Concentration in Response to Prolonged, Moderate Aerobic Exercise in Young Men and Women. Horm Metab Res. 2014; 46: 150-154.

14. Kurdiova T, Balaz M, Vician M, Maderova D, Vlcek M, Valkovic L, Srbecky M, Imrich R, Kyselovicova O, Belan V, Jelok I, Wolfrum C, Klimes I, Krssak M, Zemkova E, Gasperikova D, Ukropec J, Ukropcova B. Are Skeletal Muscle \& Adipose Tissue Fndc5 Gene Expression and Irisin Release Affected by Obesity, Diabetes and Exercise? In vivo \& in vitro studies. J Physiol. 2014; doi: 10.1113/jphysiol.2013.264655.

15. Lecker SH, Zavin A, Cao P, Arena R, Allsup K, Daniels KM, Joseph J, Schulze PC, Forman DE. Expression of the irisin precursor FNDC5 in skeletal muscle correlates with aerobic exercise performance in patients with heart failure. Circ Heart Fail. 2012; 5: 812-818.

16. Lieber RR. Skeletal muscle structure, function, and plasticity; 2nd Ed. The physiological basis of rehabilitation. MD, USA: Lippincott Williams \& Wilkins, Baltimore. 2002

17. Moraes C, Leal VO, Marinho SM, Barroso SG, Rocha GS, Boaventura GT, Mafra D. Resistance exercise training does not affect plasma irisin levels of hemodialysis patients. Horm Metab Res. 2013; 45: 900-904.

18. Norheim F, Langleite TM, Hjorth M, Holen T, Kielland A, Stadheim HK, Gulseth HL, Birkeland KI, Jensen J, Drevon CA. The effects of acute and chronic exercise on PGC-1a, irisin and browning of subcutaneous adipose tissue in humans. FEBS J. 2014; 281: 739-749.

19. Novelle MG, Contreras C, Romero-Picó A, López M, Diéguez C. Irisin, two years later. Int J Endocrinol. 2013; 2013: 746281.

20. Pekkala S, Wiklund PK, Hulmi JJ, Ahtiainen JP, Horttanainen M, Pöllänen E, Mäkelä KA, Kainulainen $\mathrm{H}$, Häkkinen K, Nyman K, Alén M, Herzig KH, Cheng S. Are skeletal muscle FNDC5 gene expression and irisin release regulated by exercise and related to health? J Physiol. 2013; 59: 5393-5400.

21. Perry CGR, Lally J, Holloway GP, Heigenhauser GJF, Bonen A, Spriet LL. Repeated transient mRNA bursts precede increases in transcriptional and mitochondrial proteins during training in human skeletal muscle. J Physiol 2010; 588: 4795-4810.

22. Pfaffl MW, Horgan GW, Dempfle L. Relative expression software tool (REST) for group-wise comparison and statistical analysis of relative expression results in real-time PCR. Nucleic Acids Res. 2002; 30: e36.

23. Raschke S, Elsen M, Gassenhuber H, Sommerfeld M, Schwahn U, Brockmann B, Jung R, Wisløff U, Tjønna AE, Raastad T, Hallén J, Norheim F, Drevon CA, Romacho T, Eckardt K, Eckel J. Evidence against a beneficial effect of irisin in humans. PLoS One. 2013; 8: e73680.

24. Roca-Rivada A, Castelao C, Senin LL, Landrove MO, Baltar J, Belén Crujeiras A, Seoane LM, Casanueva FF, Pardo M. FNDC5/irisin is not only a myokine but also an adipokine. PLoS One. 2013; 8: e60563.

25. Ruas JL, White JP, Rao RR et al. A PGC-1a isoform induced by resistance training regulates skeletal muscle hypertrophy. Cell. 2012; 151: 1319-1331.

26. Sánchez J, Nozhenko Y, Palou A, Rodríguez AM. Free fatty acid effects on myokine production in combination with exercise mimetics. Mol Nutr Food Res. 2013; 57: 1456-1467.

27. Shan T, Liang X, Bi P, Kuang S. Myostatin knockout drives browning of white adipose tissue through activating the AMPK-PGC1a-Fndc5 pathway in muscle. FASEB J. 2013; 27: 1981-1989.

28. Sharma N, Castorena CM, Cartee GD. Greater insulin sensitivity in calorie restricted rats occurs with unaltered circulating levels of several important myokines and cytokines. Nutr Metab (Lond). 2012; 9: 90.

29. Stephenson EJ, Stepto NK, Koch LG, Britton SL, Hawley JA. Divergent skeletal muscle respiratory capacities in rats artificially selected for high and low running ability: a role for Nor1? J Appl Physiol. 2012; 113: 1403-1412.

30. Swick AG, Orena S, O'Connor A. Irisin levels correlate with energy expenditure in a subgroup of humans with energy expenditure greater than predicted by fat free mass. Metabolism. 2013; 62: 1070-1073.

31. Timmons JA, Baar K, Davidsen PK, Atherton PJ. Is irisin a human exercise gene? Nature. 2012; 488: E9-10.

32. Wen MS, Wang CY, Lin SL, Hung KC. Decrease in irisin in patients with chronic kidney disease. PLoS One. 2013; 8: e64025.

33. Zhang Y, Li R, Meng Y, Li S, Donelan W, Zhao Y, Qi L, Zhang M, Wang X, Cui T, Yang LJ, Tang D. Irisin Stimulates Browning of White Adipocytes through Mitogen-Activated Protein Kinase p38 MAP Kinase and ERK MAP Kinase Signaling. Diabetes. 2013;: DB_131106. 